\title{
Machine learning for predictive maintenance of photovoltaic panels: cleaning process application.
}

\author{
Mohamed Nabti ${ }^{1, *}$, Abdelmajid Bybi ${ }^{1}$, El Ayachi Chater ${ }^{2}$, Mohammed Garoum $^{l}$ \\ 1Mohammed V University in Rabat, Higher School of Technology in Sale, MEAT, Salé, Morocco \\ 2Mohammed V University in Rabat, Higher School of Technology in Sale, LASTIMI, Salé, Morocco
}

\begin{abstract}
It is well known that the presence of dust on the surface of PV modules has a significant impact on their efficiency. Then, the consequent reduction in energy production has a non-negligible effect on the incomes. Although there is a growing need for accurate data showing where the solar arrays need maintenance in this rush for renewable energy. The purpose of this article is to introduce the research on existing photovoltaic panel maintenance solutions and introduce a new machine learning algorithm application to minimize the cleaning process of photovoltaic modules.
\end{abstract}

\section{Introduction}

In the space of an hour, the Earth receives enough sun to supply the whole world with Electricity. Then, anyone can take advantage of the sunlight abundance by utilizing solar panels. Indeed, unlike fossil fuels, which must be mined and burned, solar panels do not require a considerable amount of resources and capital. In addition, solar energy allows us to make significant savings on our electricity bills in the long term.

So far, the energy market regards photovoltaic technology as the most mature technology in the world. However, with the research and studies carried out around photovoltaic panels or cells, a new notion of "install it and forget it" has emerged because they believe that regular monitoring is not required. However, most studies do not consider the photovoltaic system as a whole. Many things can go wrong, causing actual system performance to deviate from expected performance. Thus, many developing countries in the Middle East and North Africa have chosen to adopt this technology as an alternative energy source to meet their energy needs. For instance, Morocco has participated in this development through Noor Midelt's storage. The total CSP capacity of each storage will be between 150 MW and 190 MW [1], and subcontractors will optimize the photovoltaic capacity. Besides, Noor IV is the first large-scale photovoltaic power station under construction in Morocco with a capacity of $70 \mathrm{MW}$. Unfortunately, solar energy is essential in sites, in most cases, characterized by severe weather conditions, which will increase the possibility of material degradation and cause serious problems [2,3]. The soiling effect or dusting is the consequence of dust accumulation, which is mainly a mixture of small amounts of organic particles and/or minerals from landform sediments (such as sand, clay, or eroding limestone). However, the operation and maintenance of the solar farm are critical parameters that directly affect the leveling cost of electricity. This requires planning the best cleaning strategy to overcome any drop in power production.

In this paper, we will introduce the existing solutions for the cleaning process and gaps in every solution after that a study about the need for predictive maintenance nowadays will be introduced, followed by comparing the current approaches and methods that would lead us to think of creative methods that help in raising the efficiency of cleaning.

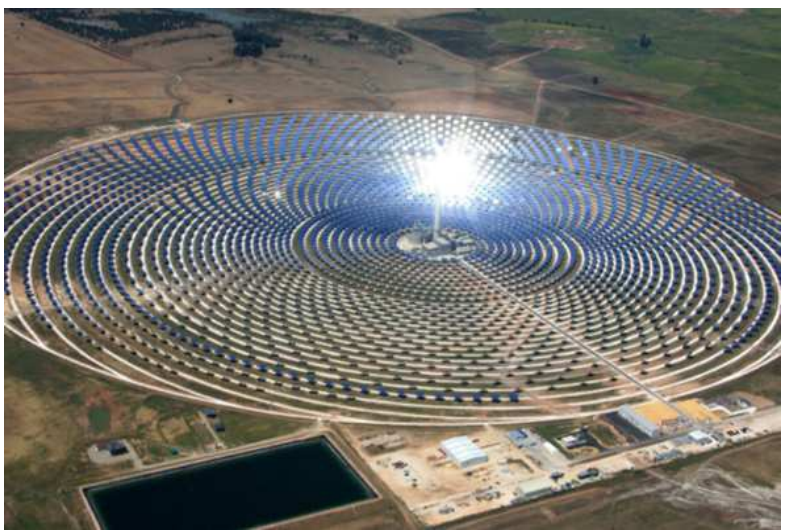

Fig. 1. Noor IV station in Ouarzazate (Morocco).[4]

\section{Problem statement}

First, there may be defects in the design and/or installation. PV modules are connected in a seriesparallel topology, where multiple panels or cells are connected in series to form a module string to obtain the best voltage. Strings of modules are then connected in parallel. Therefore, any mismatch in the string will reduce overall system performance. In addition, in many climate zones, long-term accumulation of dirt and debris on the panels can significantly reduce the performance of solar panels. Generally, drier climates leave much 
dirt, so mechanical cleaning must be performed to maintain optimal performance. This problem is not evident in areas with heavy rainfall because rainwater tends to clean solar panels. However, even in a zone with abundant rainfall, solar panels can still accumulate dirt and debris if not mounted at an angle that allows adequate water runoff. Since the panel is installed in an inaccessible location, making daily cleaning more complex, this problem is usually exacerbated.

\subsection{Existing solutions}

For the soiling effect in the PV panels, many cleaning methods use different processes and technology. For that, we cite different processes of cleaning. Starting with "Natural cleaning" of solar panels means that which occurs when the slope is steep enough for the rain to work. For the dry areas, Sprinkler systems are usually used to clean the PV modules. It has the same cleaning effect as rainwater and cleans the panel at a relatively low cost.

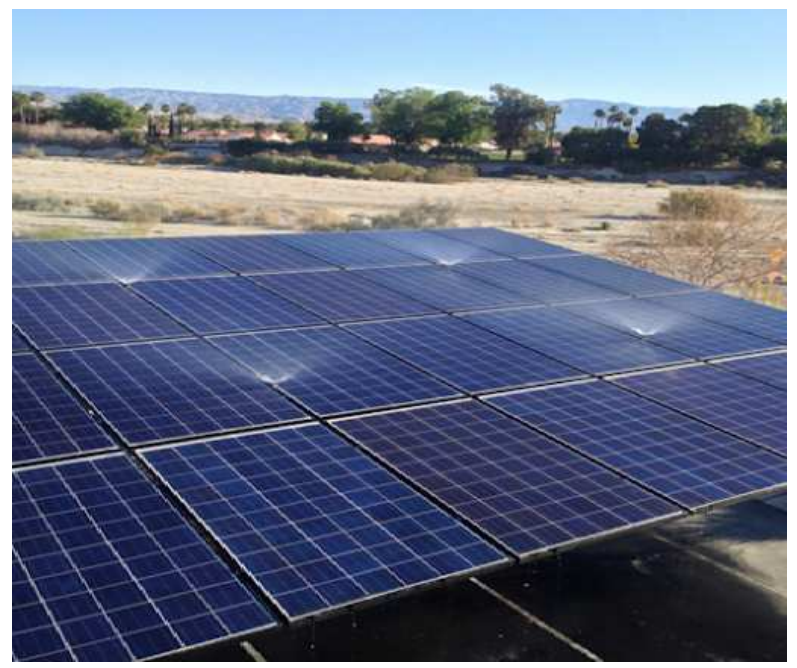

Fig. 2. Cleaning of PV panel using sprinklers. [5]

We also have the "manual cleaning" where the collaborator uses either the water or the chemical products in the dust hard to erase.

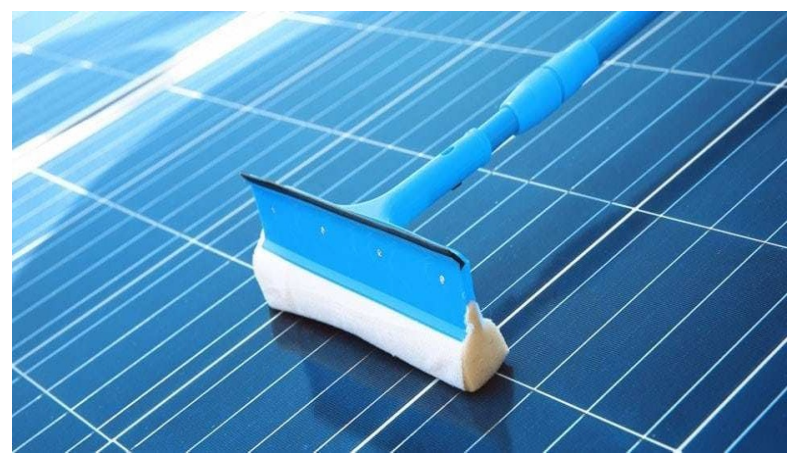

Fig. 3. Manual Cleaning of PV panels. [6]

However, cleaning solar panels is not always as straightforward. First, there is the issue of accessibility.
Because PV panels are often dangerous and challenging to reach places, it might be hard to clean them manually and take time to do it safely. However, leaving panels uncleaned might not be wise either since soiling can lead to permanent damage to the glass, limiting the installation's lifespan. Also, it is extremely common for a service provider to use a robot for cleaning.

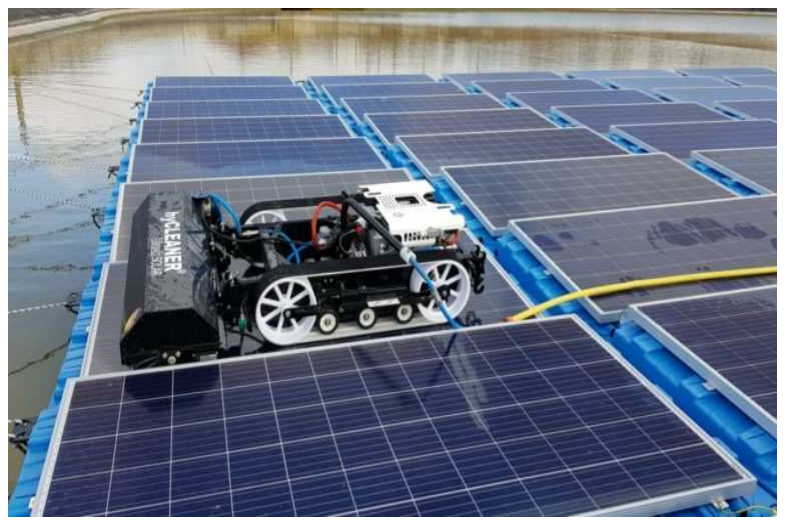

Fig. 4. Cleaning of PV panel with a robot. [7]

As the robotics field develops itself continuously lately, there are also so innovative solutions such as the drone shown in the figure.

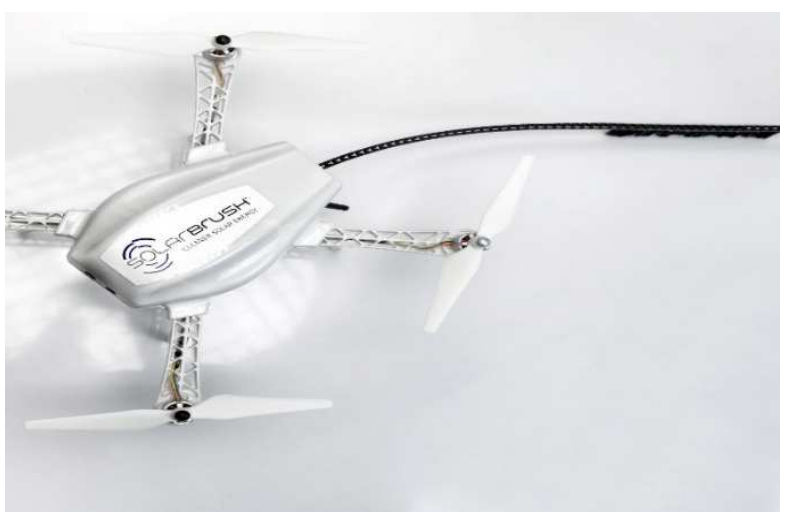

Fig. 5. Drone for the cleaning of PV Panels. [8]

The Solarbrush performs the cleaning of solar panels in a unique way, the robot performs the cleaning of photovoltaic panels Through the air. It is an unmanned aerial vehicle consisting of 4 sets of propellers and a tail. The tail has a specific material to clean the panel. The only part of the drone that is in contact with the surface of the photovoltaic panel is its tail. According to its developers, this is the main advantage of using the robot, because using this function can reduce the chance of damaging the photovoltaic panel.

Finally, of course we can't talk about robotics in PC modules cleaning without mentioning Ecoppia E4, as can be seen in figure. 


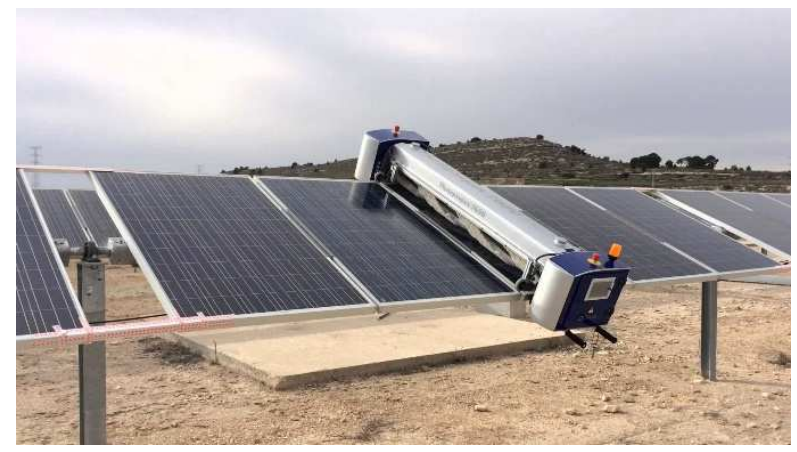

Fig. 6. Close view of Ecoppia E4. [9]

A fully autonomous robot uses microfiber brushes to sweep the dust from the panels. It is designed for large rows of panels located in dry and sandy environments. The robot uses a photovoltaic panel and battery on board to store energy, this allows the robot to perform cleaning at night and the positioning of the photovoltaic panel at the bottom of the rail allows it to be cleaned by the robot itself.

\subsection{Gaps in the existing solutions}

Although these solutions have been practiced in the market and their effectiveness is beyond doubt, the system must provide proper cleaning conditions for dirt, not obstruct the incidence of solar radiation, and will not affect the photovoltaic modules in the process. In fact, "Natural cleaning" is never enough. Rainwater, loaded with mineral salts, causes the appearance of lichen and moss. For the sprinkles approach, water quality should be tested, and water should be selected For places with untreated water, treatment is recommended. Although this is a better working system, there are Some disadvantages such as the quantity of water

While "manual cleaning" represents a risk for workers who often work at the height of about 20 meters, sometimes, from the ground, with a risk of falling. Besides, chemicals can be aggressive to solar panel materials, reducing their transparency. In addition, Chemical residues from cleaning the photovoltaic system cannot be dumped into the ground but must comply with environmental regulations. Preserving the environment has become a decisive element in new cleaning methods. Finally, most of the robots used in the cleaning are too heavy compared to the panel manufacturer's recommendations, which generates cracks, which are not necessarily visible but very real.

\subsection{Summary}

Given the gap and the size of the problem in each existing solution, we propose a new interface that plays the role of predictive maintenance of system-level photovoltaic systems to predict the correct way to clean photovoltaic installations. This maintenance tool helps to optimize the return on investment and minimize the warranty time.

\section{Materials and methods}

Considering that this article provides a brief and easy-to-understand overview of PV predictive maintenance, the literature review has two essential parts. First, provide justifications for PV predictive maintenance needs. Second, provide current methods and opportunities for predictive maintenance of photovoltaics, and then the purpose of the paper derived from this research.

\subsection{Need for predictive maintenance}

Although most of the literature documents the benefits of PV because it is related to low maintenance costs, anecdotal evidence and recent research report a different story, and it is used in real-world nonlaboratory applications. As stated, the demand for cheap renewable energy sources is at the highest that it has ever been. Therefore, solar arrays and installations have been being erected the world over. There is a growing need for accurate data showing where the solar arrays need maintenance in this rush for renewable energy.

Although rare in the literature, few studies provide specific examples of photovoltaic systems that require maintenance. For instance, in [10], the authors conducted a study showing the effectiveness of modeling a system to analyze the sensitivity of soiling effect on the energy production of the PV panels by collecting the data of the weather and comparing the energy production expected and the real one to detect the essential factor impacting the PV installation. They came to the conclusion that the most influential the relative humidity followed by wind direction, wind speed, solar irradiance, ambient temperature, and rainfall.

Then, in [11], the published study evaluated the ability of a new method that uses Brewster's angle to evaluate the intensity of light reflected on glass surfaces of different densities, thereby quantifying the drop in glass transmittance. The results showed that the intensity of the reflected ray using Brewster angle decrease with the increase of the density of deposition on the surface of the glass sample.

\subsection{Current approaches and methods}

Due to the increase in the installation of photovoltaic systems, the demand for quality control for testing and verification continues to rise. Several known methods for assessing the potential performance degradation of photovoltaic systems, including visual inspection of the system and individual components, analysis of curves and insulation resistance of photovoltaic panels, photovoltaic panels, Infrared thermal imaging, and calculate and compare the estimated actual power generation. The first three can be summarized as manual diagnoses [12-13]. The latter can be broken down into failure mode and impact analysis methods, machine learning and prediction, and real-time sensor analysis. 
Manual diagnosis has the lowest cost but the lowest detection accuracy. Machine learning and prediction are moderately costly and provide an average detection accuracy [14-15]. Real-time sensors are the most expensive but provide the highest detection accuracy [16-17]. Figure 7 is an illustration about the current approaches cited earlier contaitining a comparison of the cost and and detection accuracy of each of the three approved approaches [18].

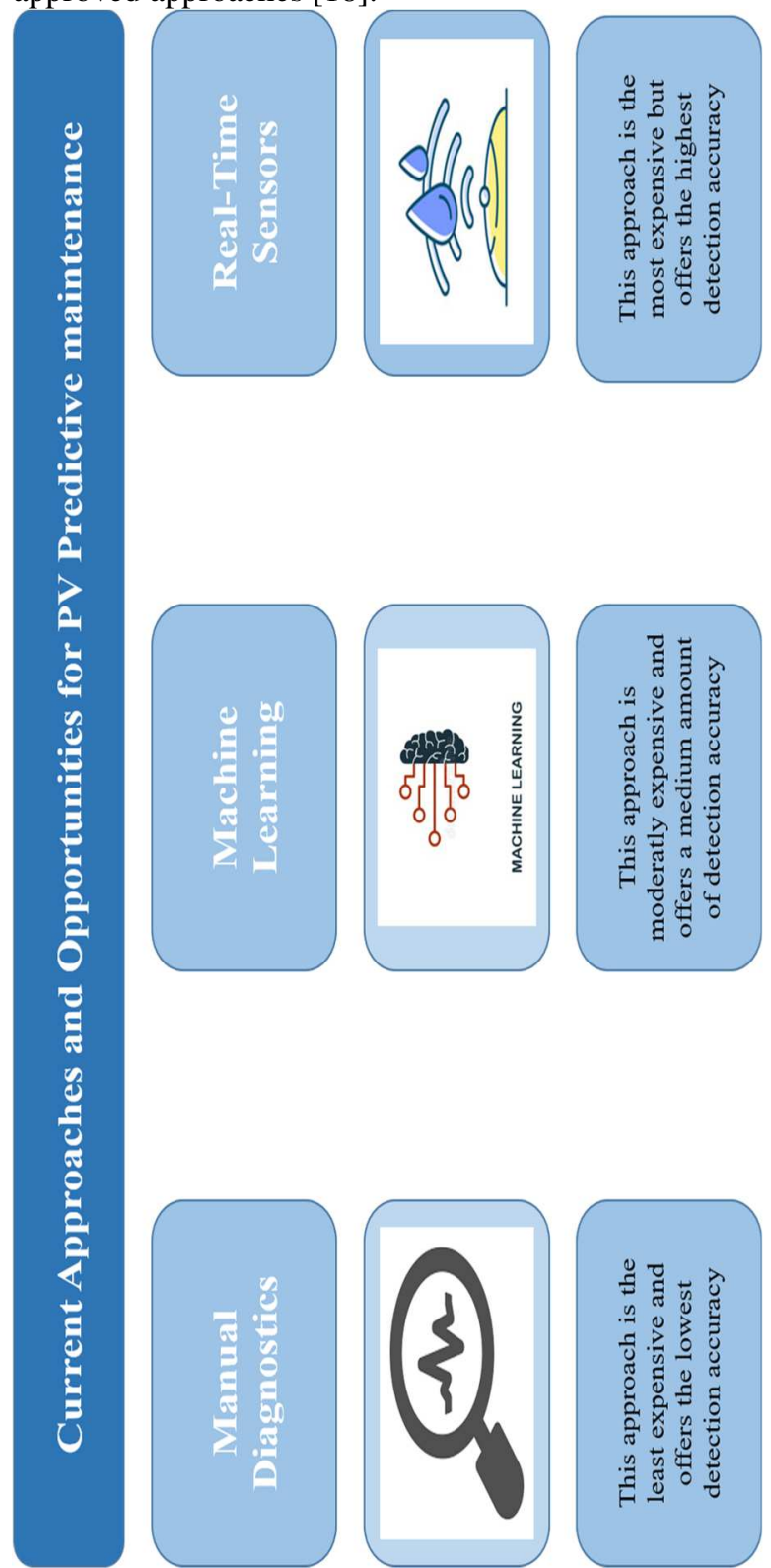

Fig. 7. Current Approaches of PV Predictive maintenance.

\subsection{Purpose of the review}

Taken into account that the current solutions for the maintenance of PV panels are laking and that the predictive maintenance can bring many benefits, this paper aim to propose a solution for the cleaning as an output of the predictive maintenance machine learning's algorithm based on the type and source of the soiling effect. Figure 8 represents a practical method of analysing the inputs to give a significant output to analyse by using the samples recorded in comparing the target output with the predicted output using the same samples.

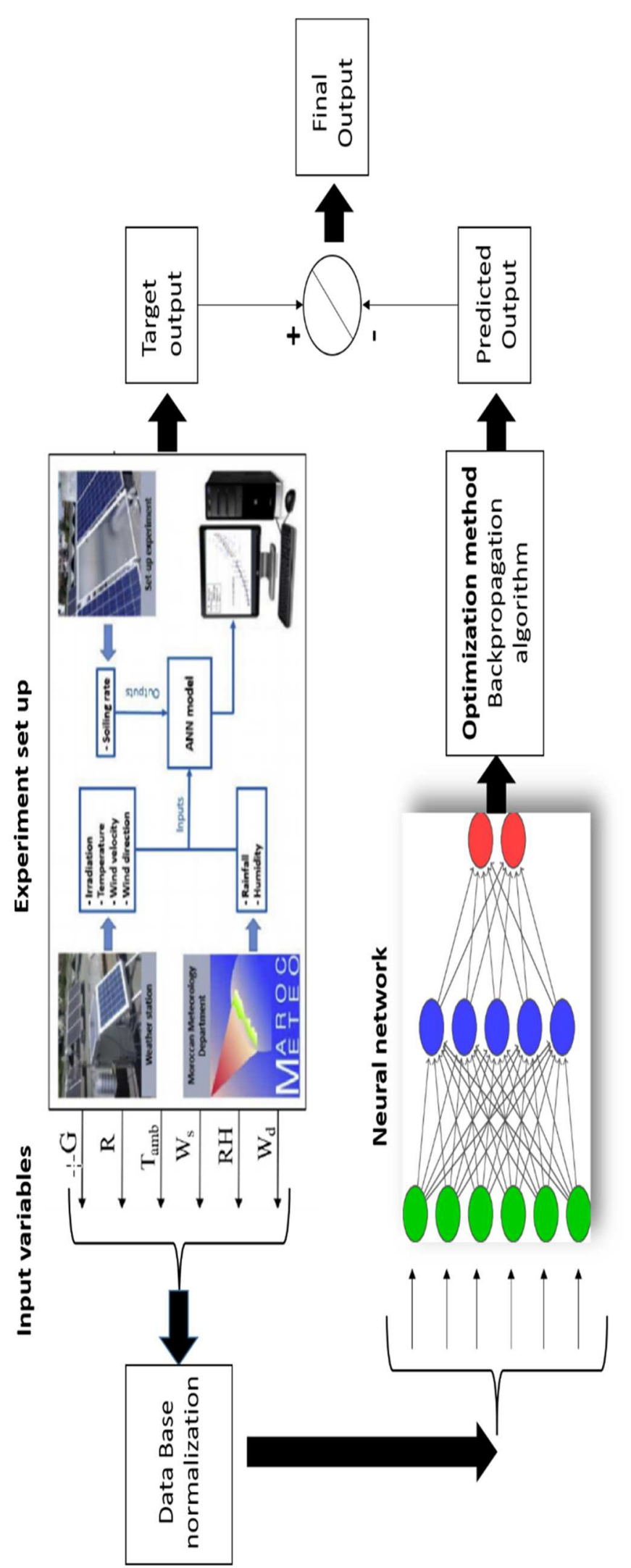

Fig. 8. Process of analyzing the data. [10] 


\section{Conclusion}

As efficiencies related to solar production and distribution increase, individuals/companies have also assumed the role of energy producers. However, few resources are dedicated to understanding and optimizing the long-term performance of photovoltaic systems. Designing methods and tools to ensure the regular operation of solar systems will positively impact photovoltaic system owners (including utility companies). Specifically, in this research, we tried to improve the priority of photovoltaic system error detection and predictive maintenance. Ultimately, we recommend that $\mathrm{PV}$ system owners need a new process in the PV system predictive maintenance tool to optimize return-on-investment and minimize warranty claims time. The tool should monitor incoming solar irradiance and weather factors to determine real-time actual power generation and compare it with the manufacturer's warranty.

However, many other research areas can and should be targeted. Future research should continue to investigate, assess, and evaluate the long-term quality of the PV systems.

This analysis can be implemented in other artificial intelligence-based renewable energy models to complete the hollow knowledge left by using these technologies in the physical understanding of phenomena. Finally, this method is a promising framework that can be used for intelligent sensors in photovoltaic module cleaning systems to improve their operating efficiency.

\section{References}

1. Alae, Azouzoute, and Ahmed, Alami Merrouni, and Mohammed, Garoum, and El Ghali, Bennouna. (2019) "Soiling Loss of Solar Glass and Mirror Samples in the Region with Arid Climate".

2. Bouraiou, A.; Hamouda, M.; Chaker, A.; Neçaibia, A.; Mostefaoui, M.; Boutasseta, N.; Ziane, A.; Dabou, R.;Sahouane, N.; Lachtar, S. Experimental investigation of observed defects in crystalline silicon PV modules under outdoor hot dry climatic conditions in Algeria.

3. Klugmann-Radziemska, E. Degradation of electrical performance of a crystalline photovoltaic module due to dust deposition in northern Poland.

4. https://chantiersdumaroc.ma/constructiondurable/premiere-mondiale-noor-iv/ access : 9 august 2020

5. http://energy.asu.edu.jo/index.php/r-d/solar-panelcleaning-system access : 9 august 2020

6. https://ecotality.com/how-to-clean-solar-panels/ access : 10 august 2020 .

7. https://hycleaner.eu/en/produkte/hycleaner-blacksolar/ access : 9 august 2020

8. https://www.imnovation-hub.com/science-andtechnology/drones-new-generation-solar-farmers/ access : 9 august 2020
9. https://www.technocrazed.com/fully-automatedrobotic-cleaning-makes-solar-panels-moreefficient/closer-view-of-ecoppia-e4 9 august 2020 10. B. Laarabi, O. May Tzuc, D. Dahlioui, A. Bassam, M. Flota-Banuelos, A. Barhdadi: Artificial neural network modeling and sensitivity analysis for soiling effects on photovoltaic panels in Morocco.

11. Alae Azouzoute, Mostafa Chouitar, Mohammed Garoum, El Ghali Bennouna1, Abdellatif Ghennioui : A New PV Soiling Monitoring Device for Optimized Cleaning Strategy.

12. Buerhop-Lutz and Scheuerpflug (2015): Inspecting PV-plants using aerial, drone-mounted infrared thermography system. 3rd Southern African Solar Energy Conference, Kruger

National Park, South Africa, 11-13 May 2015

13. Zamora, R.J.; Dutton, E.G.; Trainer, M.; McKeen, S.A.; Wilczak, J.M.; Hou, Y.-T : The accuracy of solar irradiance calculations used in mesoscale numerical weather prediction.

14. Marquez and Coimbra: Forecasting of global and direct solar irradiance using stochastic learning methods, ground experiments and the NWS database.

15. Alessandro Betti, Maria Luisa Lo Trovato, Fabio Salvatore Leonardi, Giuseppe Leotta, Fabrizio Ruffini , and Ciro Lanzetta : PREDICTIVE MAINTENANCE IN PHOTOVOLTAIC PLANTS WITH A BIG DATA APPROACH.

16. Moreno-Garcia, I.M.; Palacios-Garcia, E.J.; Pallares-Lopez, V.; Santiago, I.; Gonzalez-Redondo, M.J.;Varo-Martinez, M.; Real-Calvo : Real-time monitoring system for a utility-scale photovoltaic power plant

17. Adhya, S.; Saha, D.; Das, A.; Jana, J.; Saha : An IoT based smart solar photovoltaic remote monitoring and control unit. 2nd International Conference on Control, Instrumentation, Energy \& Communication (CIEC), Kolkata, India, 28-30 January 2016; pp. 432436.

18. BOSMAN, Lisa B., LEON-SALAS, Walter D., HUTZEL, William, et al. PV System predictive maintenance: Challenges, current approaches, and opportunities. Energies, 2020, vol. 13, no 6, p. 1398' 